\title{
Silicon-based Quantum Computation
}

\author{
B. E. Kane* \\ Laboratory for Physical Sciences \\ University of Maryland \\ College Park, MD 20740 USA
}

(February 1, 2008)

\begin{abstract}
An architecture for a quantum computer is presented in which spins associated with donors in silicon function as qubits. Quantum operations on the spins are performed using a combination of voltages applied to gates adjacent to the spins and radio frequency magnetic fields applied resonant with spin transitions. Initialization and measurement of electron spins is made by electrostatic probing of a two electron system, whose orbital configuration must depend on the spin states of the electrons because of the Pauli Principle. Specific devices will be discussed which perform all the necessary operations for quantum computing, with an emphasis placed on a qualitative presentation of the principles underlying their operation.

The likely impediments to achieving large-scale quantum computation using this architecture will be addressed: the computer must operate at extremely low temperature, must be fabricated from devices built with near atomic precision, and will require extremely accurate gating operations in order to perform quantum logic. Refinements to the computer architecture will be presented which could remedy each of these deficiencies. I will conclude by discussing a possible specific realization of the computer using $\mathrm{Si} / \mathrm{Si}_{x} \mathrm{Ge}_{1-x}$ heterostructures into which donors are deposited using a low energy focused ion beam.
\end{abstract}

\section{INTRODUCTION}

One of the greatest challenges facing physics at the onset of the twenty-first century is to determine whether or not construction of a large-scale quantum computer is possible both in principle and in terms of practical technical issues. While quantum algorithms have been developed which prove convincingly that quantum computers have capabilities conventional computers cannot duplicate, future applications of these hypothetical machines remain mostly a subject of speculation. It seems likely, however, that if a large-scale quantum computer can be built, then these machines will have a significant impact in the coming century, when information processing technology will only be more important than it is today.

Large-scale conventional computation only became possible when thousands of transistors could be integrated onto a single solid state chip, and it is widely believed that scalable quantum computation will be achieved when solid state quantum logical devices are similarly integrated. It should be stated at the outset, however, that since the likely impediments to large-scale quantum computation will be altogether different from those that faced conventional computers, scaling a solid state quantum computer may not prove to be viable. Ultimately the only test of viability of any quantum computer architecture must come from the laborious development and refinement of experimental candidate devices and architectures.

While by no means assured of success, quantum computer architectures implemented in solids, and especially in semiconductors, have the potential to take advantage of the enormous amount of ingenuity and resources that have gone into the development of contemporary microelectronics. This development will no doubt continue until electronic device sizes approach atomic dimensions, a realm in which a wide variety of device concepts potentially capable of quantum computation will be possible to implement.

Spins are perhaps the ideal qubits embodied in natural systems, and several candidate quantum computer architectures have been developed with spin qubits embedded in solid state materials [1] [2] [3] [4] [5] [6]. The essence of this author's published proposal for a solid state quantum computer [3] is that spins associated with donors in silicon - the semiconductor that is the mainstay of conventional computer technology - are ideally suited to function as qubits in a quantum computer. This fact is a consequence of the high degree of isolation of electron and nuclear spins at donors in Si from their surroundings at low temperatures $(T<4 \mathrm{~K})$. This isolation means that the highly coherent manipulations of the spins necessary for quantum computation are possible if there is a suitable means of controlling the interactions of individual spins with each other and with externally applied fields. In the proposal [3], control of individual spin dynamics is achieved by applying voltages to metal gates, located adjacent to the donors. The donors must be in prescribed locations within the silicon host to enable accurate gate control, a requirement which will pose perhaps the most significant obstacle to the implementation of the computer design.

Readout of single spin qubits (and also initialization of the qubits) is achieved by a two step process: first, a spin quantum number is transferred to a property of the charge configuration of a system. Second, the charge configuration is determined using sensitive electronic de- 
vices. Spin quantum numbers can most easily affect the charge configuration of a two electron system: the Pauli Exclusion Principle requires that two electrons can only occupy the same orbital state if they are in a mutual spin singlet state, with spins pointing in opposite directions. Sensitive electrometers now have resolution much better than a single electron charge, and are thus capable of determining whether two electrons are in a singlet or a triplet state (when the two spins are pointing in the same direction and the electrons cannot occupy the same orbital state).

The computer is capable of performing both logic and measurement operations in parallel and is thus compatible with error correcting algorithms, which will be an inevitable attribute of any large-scale quantum computer. The high degree of isolation of spins in Si means that performing logical operations with the accuracy required $\left(\leq 10^{-4}\right)$ for error corrected continuous computation [7] [8] is in principle possible.

The architecture is not without its deficiencies, and subsequent to the publication of the original proposal several suggestions were made that could lead to improvements of the computer design [9], [10], [5]. Consequently, I will address several specific modifications that will likely need to be incorporated into the architecture if it is to be scaled: firstly, I will discuss techniques that would allow the computer to operate at higher temperatures by refrigerating spins on the computer chip. Secondly, I will describe possible ways in which free electrons can be used to transmit quantum information across large distances on the computer chip [9], making the execution of quantum algorithms more efficient. Finally, I will discuss approaches to quantum logic that are insensitive to the inevitable fluctuations present in solid state devices [10].

I will conclude by presenting a specific material and fabrication technology for the computer: high quality $\mathrm{Si} / \mathrm{Si}_{x} \mathrm{Ge}_{1-x}$ heterostructures grown by molecular beam epitaxy into which single donor ions are deposited during heterostructure growth 5 . The devices and fabrication technologies presented, however, are not intended to be a blueprint for the construction of a quantum computer but rather are an effort to stimulate research and thinking both in the nanostructure and device physics community, as well as in the community focused on theoretical issues of quantum computation and measurement.

\section{ELECTRON AND NUCLEAR SPINS AT DONORS IN SILICON}

For crystals of pure $\mathrm{Si}$ at low temperatures $(T)$, no electrons are in the conduction band, and the material is an insulator. The addition of Column $\mathrm{V}$ donors to the Si crystal results in electron states near in energy to the conduction band but weakly bound to the donor sites at low $T$. The theory of these weakly bound states was
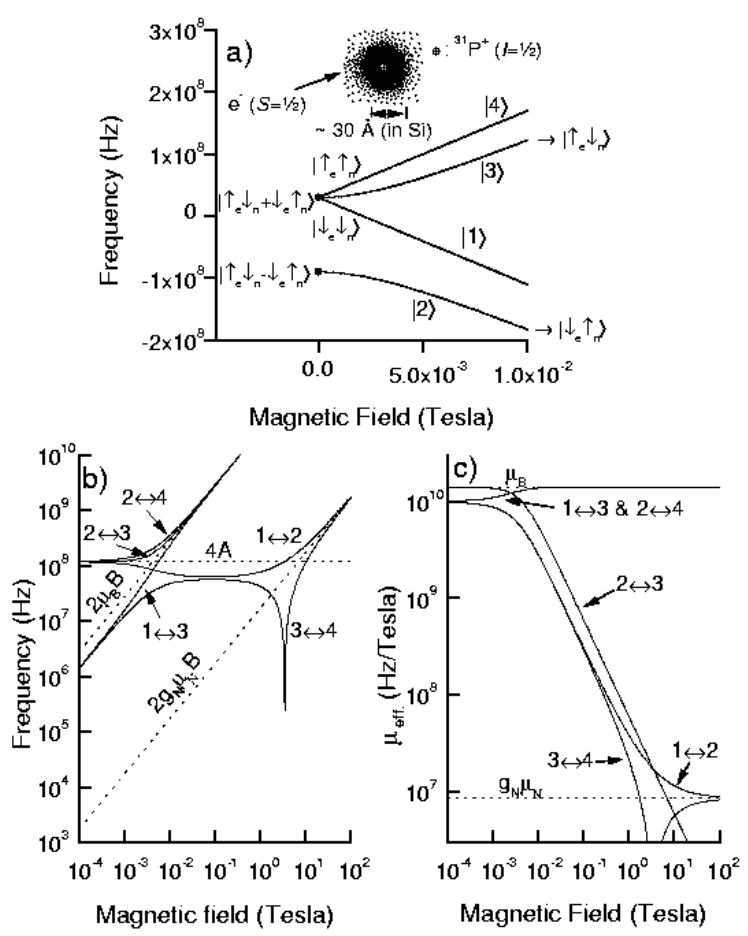

FIG. 1. (a) Spin energy levels of $\mathrm{a}^{31} \mathrm{P}$ donor in $\mathrm{Si}$ and (b) energy differences of the levels as a function of applied magnetic field, $B$. At $B=0$ the electron-nuclear spin singlet is 120 $\mathrm{MHz}$ below the degenerate triplet states. As $B$ is increased these energy differences ultimately approach the electron and nuclear Zeeman energies. (c) The matrix elements coupling the different levels as a function of $B$. Coupling between states with different nuclear spin decreases with increasing $B$, and consequently operation of a nuclear spin quantum computer is potentially more rapid at smaller magnetic fields.

developed in the fifties 11]; the electron states resemble those in a hydrogen atom, but with an expanded Bohr radius $\left(a_{B} \cong 15-30 \AA\right)$ and a reduced binding energy $\left(E_{b} \cong\right.$ $10-50 \mathrm{meV})$.

The electron has spin $S=1 / 2$, and all Column V donors have nonzero nuclear spin $I$. (In contrast all Group IV elements, including $\mathrm{Si}$, have stable isotopes with $I=0$, which means nuclear spins can in principle be entirely eliminated from these materials by isotope refinement.) The simplest system - and the one that has been most exhaustively studied - is $\mathrm{P}$ doped $\mathrm{Si}$, in which $I=1 / 2$. The spin Hamiltonian for the nucleus-electron system in $\mathrm{Si} P$, with magnetic field $B \| z$ is:

$$
H_{e n}=\mu_{B} B \sigma_{z}^{e}-g_{n} \mu_{n} B \sigma_{z}^{n}+A \sigma^{e} \cdot \sigma^{n},
$$

where $\sigma$ are the Pauli spin matrices (with eigenvalues $\pm 1), \mu_{B}$ and $\mu_{n}$ are respectively the Bohr and nuclear 
magneton, $g_{n}$ is the nuclear g-factor $\left(=1.13\right.$ for $\left.{ }^{31} \mathrm{P}\right)$, and $A=\frac{8}{3} \pi \mu_{B} g_{n} \mu_{n}|\Psi(0)|^{2}$ is the contact hyperfine interaction energy, with $|\Psi(0)|^{2}$ the probability density of the electron wave function evaluated at the nucleus [12]. For electrons in $\mathrm{Si},|g-2| \cong 10^{-3}$, and consequently $g=2$ is assumed in Eq. 1. Because the electron wave function is strongly peaked at the donor site, the contact hyperfine interaction energy of the electron and the donor nucleus greatly exceeds dipolar spin interactions. The Hamiltonian (11) can readily be solved exactly [13] and the energy levels for Si:P are plotted in Fig. 1 as a function of $B$.

At $B=0$ energy eigenstates of Eq. 1 are also eigenstates of the spin exchange operator. The ground state is the singlet $\left(\left|\uparrow_{e} \downarrow_{n}-\downarrow_{e} \uparrow_{n}\right\rangle\right)$, lying $4 A(=120 \mathrm{MHz}$ in Si:P [14]) below the threefold degenerate triplet excited states. Application of $B$ leads to evolution of the states into well defined electron and nuclear spin states as first the electron and then the nuclear Zeeman energies exceed $4 A$ (Fig. 1b). Transitions between the different energy levels are induced by a radio frequency magnetic field $B_{r f}$ applied at a frequency resonant with the energy level difference. The magnitude of the matrix element $\mu_{\text {eff }}$. coupling between the levels is plotted in Fig. 1c. At $B=0$, couplings for allowed transitions are approximately the same as for an uncoupled electron spin, $\mu_{B}$. As $B$ is increased and electron and nuclear transitions become distinct, the coupling between the nuclear states weakens, approaching $\mu_{n}$ at large $B$.

There are several reasons why the coupled electronnucleus system at donors in $\mathrm{Si}$ is an excellent building block for quantum computational devices: first, the transition times between the states depicted in Fig. 1 can be exceedingly long at low temperatures and are of order one hour at $T=1 \mathrm{~K}$ and $B=0.1 \mathrm{~T}$ [15] 16]. Secondly, the linewidths of the transitions are very narrow and are limited by the small percentage of ${ }^{29} \mathrm{Si}$ nuclei with $I=1 / 2$ present in the Si crystal that also interact with the electron via hyperfine interactions 115 . In isotopically purified ${ }^{28} \mathrm{Si}$ (with $I=0$ ), Si:P linewidths are $<1 \mathrm{MHz}[15$. The most relevant factor for qubits is the phase relaxation time, $t_{\phi}$. In isotopically purified $\mathrm{Si}: \mathrm{P}, t_{\phi}$ exceeds $0.5 \mathrm{msec}$ [17] [18], and is probably limited by dipolar interactions between the electron spins. In a quantum computer dipolar interactions can be eliminated as a source of decoherence using compensating algorithms [19].

\section{QUANTUM OPERATIONS WITH THE Si:P SYSTEM}

The Si:P system is a natural two qubit quantum computer [20]: the controlled NOT operation, in which an electron spin flip occurs conditioned on the state of the nuclear spin, is performed by exciting the transition $\left|\downarrow_{e} \uparrow_{n}\right\rangle \leftrightarrow\left|\uparrow_{e} \uparrow_{n}\right\rangle$, for example. The SWAP operation is performed by exciting the transition $\left|\downarrow_{e} \uparrow_{n}\right\rangle \leftrightarrow\left|\uparrow_{e} \downarrow_{n}\right\rangle$.

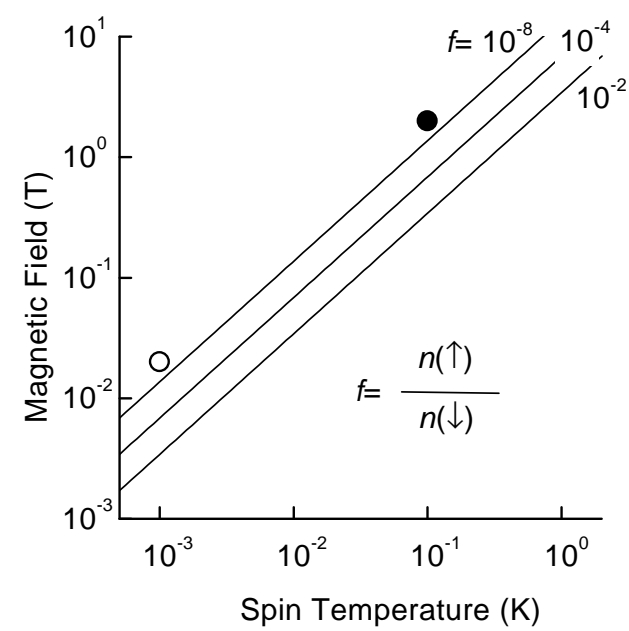

FIG. 2. Contours of equilibrium electron spin polarization plotted as a function of $B$ and temperature $T$. Extremely high spin polarization requires a combination of very low $T$ and large $B$. While $T=100 \mathrm{mK}$ and $B=1 \mathrm{~T}$ (filled circle) may readily be obtained in the laboratory, operation at lower magnetic fields and lower spin temperatures may be possible (open circle) if on-chip spin refrigeration devices can be developed.

The electron and nuclear spins have distinct characteristics that are favorable for quantum computation. The electron spins are de-localized and mobile, and they can couple to additional nuclei via the hyperfine interaction or to other electrons via the exchange interaction. The location of the electron can be controlled by electric fields applied to gates on the Si surface, as is done in conventional Si devices. Electron spins can also in principle be measured using device concepts based on the Pauli Principle. The nuclear spins are much more weakly coupled to the environment, have no orbital degrees of freedom, rotate much more slowly in an applied $B$ than electron spins, and are consequently almost ideally suited to function as qubits in a quantum computer.

While a quantum computer architecture has been proposed based on donors in silicon-germanium heterostructures which uses exclusively the electron spins [5], no common donor exists for these semiconductors that does not possess a nuclear spin. Consequently, it is preferable to adopt an architecture which uses the properties of both the electron and nuclear spins to advantage. In the architecture to be presented here, nuclear spins are the qubits and quantum memory of the computer, while the electrons are used to mediate interactions between nuclear spins. Qubit initialization and readout is performed by a combination of transfer of spin between nuclear and electron spins (for example by using the SWAP operation) and measurement operations on the electron spins. 


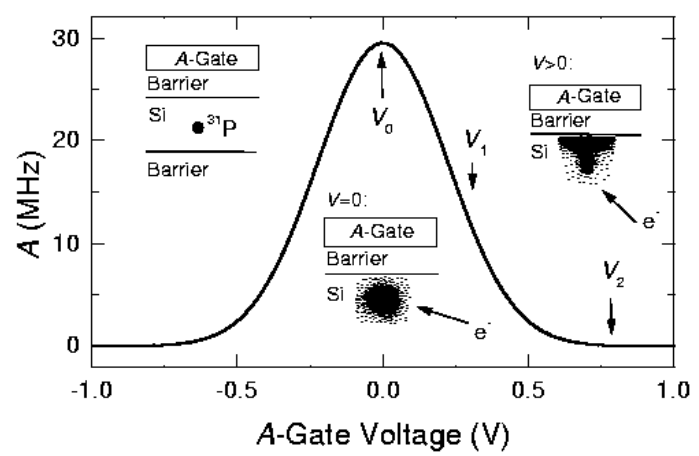

FIG. 3. An $A$-gate is located above $\mathrm{P}$ donors in $\mathrm{Si}$ and controls the hyperfine interaction between the donor nucleus and the surrounding electron by distorting the electron wave function. The plot is a qualitative depiction of $A$ as a function of gate voltage. $A$ is least sensitive to gate voltage fluctuations when $d A / d V=0$ (at $V_{0}$ and $V_{2}$ ) and most sensitive to voltage fluctuations near $V=V_{1}$.

\section{ENVIRONMENT NECESSARY FOR QUANTUM COMPUTATION}

As was mentioned above, the long relaxation times of Si:P necessary for quantum computation only occur at $T \leq 4 \mathrm{~K}$. At higher temperatures phonon scattering rapidly decreases the relaxation times [15] [16]. A more stringent requirement placed on the environment necessary for quantum computation is that the spins must be highly polarized. The equilibrium polarization of electrons as a function of $T$ and $B$ is plotted in Fig. 2. Strong polarization of the electrons requires both high magnetic fields $(B \simeq 1 \mathrm{~T})$ and very low temperatures $(T \simeq 0.1 \mathrm{~K})$. These conditions were assumed in the original quantum computer proposal and are readily obtainable in low temperature experimental laboratories. The extremely long relaxation times of the spins imply that nonequilibrium polarizations of the spins are possible in less extreme environments, achieved for example by injection of electrons from ferromagnetic contacts or by optical pumping. I will discuss below a third alternative for polarizing electron spins by using devices which perform spin refrigeration. These ideas, if implemented, would enable a spin quantum computer to operate in the more favorable environment of higher temperatures and lower magnetic fields.

\section{GATE-CONTROLLED QUANTUM LOGIC}

The Si:P two qubit quantum computer cannot be integrated into a large-scale quantum computer unless quan- tum operations can be applied selectively to particular spins (bulk spin resonance operations perform identical operations on all spins in the system). Also, connectivity must be established between spins in order to do arbitrary multi-qubit quantum logic. Both selectivity and connectivity can be achieved by applying electric fields to metal gates adjacent to the spins. An approach to selective single spin quantum logic is shown in Fig. 3. A metal "A-gate" is located directly above a donor site, separated by a barrier from the Si semiconductor material (possible materials that could be used for the barrier will be discussed below). Application of a voltage bias to the gate creates an electric field which distorts the electron wave function surrounding the donor [21]. This distortion changes the electron density at the donor nuclear site and also the hyperfine interaction energy $A$. Because the energy spacings of the spin levels are sensitive to $A$, an $A$-gate can selectively bring a single $\mathrm{Si}: \mathrm{P}$ system into (or out of) resonance with a globally applied external $B_{r f}$. Single spin quantum logic can thus be performed only at selected sites.

The simplest way to couple additional spins into a quantum computer architecture is to fabricate an array of Si:P sites in close proximity (Fig. 4). As the site separation becomes comparable to the Bohr radius, the electron wave functions begin to overlap. Tunneling of electrons between sites then becomes possible, leading to an exchange interaction between the electron spins and also to an indirect (or electron mediated) exchange interaction between the nuclear spins. For the case when the two electron spins are in the $|\downarrow \downarrow\rangle$ state and are each coupled to the donor nuclear spins by the same hyperfine interaction energy $A$, the nuclear spin exchange frequency is approximately:

$$
h \nu_{J}=2 A^{2}\left(\frac{1}{\mu_{B} B-2 J}-\frac{1}{\mu_{B} B}\right)
$$

where $J$ is the electron spin exchange energy, and $2 J<$ $\mu_{B} B$ is assumed. The magnitude of $J$ between electron spins on donors as a function of their separation $r$ can be approximated from equations derived for the case of well- separated $\mathrm{H}$ atoms [22]:

$$
J(r) \sim E_{b}\left(\frac{r}{a_{B}}\right)^{\frac{5}{2}} \exp \left(\frac{-2 r}{a_{B}}\right) .
$$

This function, with values appropriate for $\mathrm{Si}$, is plotted in Fig. 5. Substantial nuclear spin exchange between donors requires that $J$ and $\mu_{B} B$ must be comparable, and consequently the donors and gates must be spaced of order $100 \AA$ apart, a scale which is near the limit of current nanofabrication technology.

Because exchange interactions depend on the overlap of the electron wave functions, they can be effectively controlled by a voltage bias applied to a " $J$-gate" lying between donor sites (Fig. 4). Exchange interactions lead 


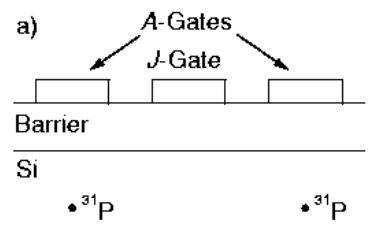

b)

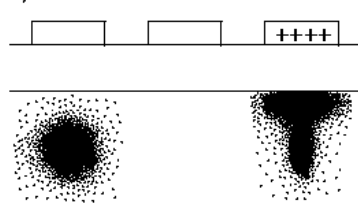

c)

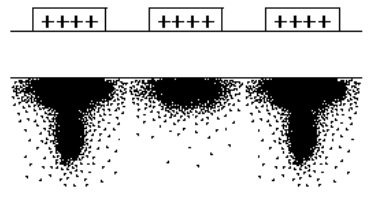

FIG. 4. (a) Configuration of gates and two donors for doing one and two qubit logical operations: in addition to $A$-gates above the donors, $J$-gates lie between donor sites. (b) One qubit logical operations are performed by applying an $A$-gate bias which brings a selected spin into resonance with an external rf magnetic field. (c) Two qubit operations are performed by lowering the potential barrier between donor sites with the $J$-gate and turning on exchange coupling between the donors. Electron mediated nuclear spin exchange will then occur between the donor nuclei.

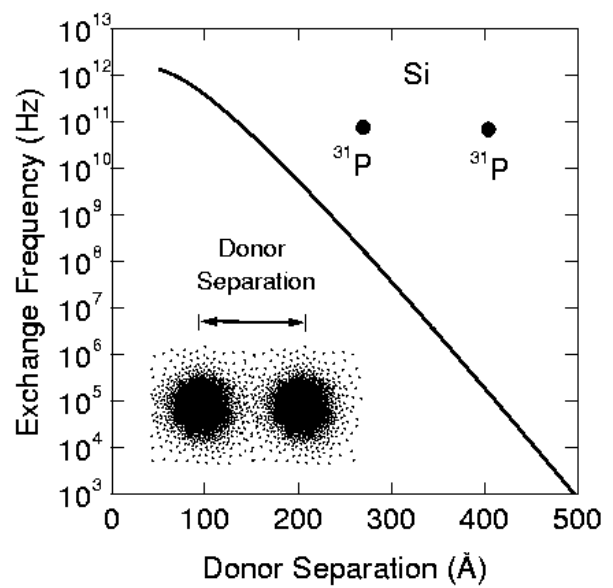

FIG. 5. The strength of the electron exchange coupling between donor sites, plotted as a function of their separation. The exchange coupling must be comparable to the electron Zeeman energy for nuclear spin exchange to occur between sites. This requirement means the donor spacing must be of order $100 \AA$.

naturally to the SWAP operation of quantum logic, in which the spin quantum numbers of two qubits are interchanged. The SWAP operation in combination with single spin operations can be used as the primitive operations of a universal quantum computer [1]. In the configuration shown in Fig. 4, a negative voltage bias applied to the $J$-gate decouples the adjacent spins. A positive voltage applied to the gate turns on the exchange interaction between the spins. A SWAP operation is performed if the voltage pulse has the appropriate length. Calculating the value of the exchange interaction as a function of separation between donors and of gate voltage in realistic device configurations is difficult and is the subject of current research 23] [24]. An added complication in Si is that the degenerate band structure leads to oscillatory behavior of the exchange interaction due to interference between electrons in different conduction band minima 25.

\section{SPEED OF COMPUTER OPERATION}

The speed of the computer architecture described above is limited by the rate at which single spin operations can be performed. This rate is approximately the product of $B_{r f}$ and $\mu_{e f f}$, plotted in Fig. 1c. $B_{r f}$ cannot be too large or else it will excite transitions between states not resonant with the field. Additionally, large values of $B_{r f}$ will introduce eddy current heating of conductors (gate leads for example) in the neighborhood of the computer. A reasonable value of $B_{r f}$ is $10^{-3} \mathrm{~T}$. This 
value, and a $\mu_{\text {eff. }}$ appropriate for nuclear spin flips at $B=1 \mathrm{~T}$, yields a single spin operation rate of $r \approx 10-100$ $\mathrm{kHz}$.

The speed of two spin operations will depend on the strength of the exchange coupling between sites, but can be comparable to or greater than the rate for single spin operations. More relevant is that spins can only interact with their neighbors, so that many SWAP operations must be performed to do a two spin operation on two spins that are far apart.

It is certainly possible to find alternative systems in which gate operations could be performed much more rapidly than the system discussed above. The stringent requirements on the accuracy of logical operations in a quantum computer are likely to be more easily fulfilled in systems with slow dynamics, so the slowness of logical operations of the nuclear spin quantum computer is not necessarily a disadvantage. Clearly, it is the ability to perform quantum algorithms, not the clock speed, which will distinguish any quantum computer from its classical counterparts.

\section{NOISE INTRODUCED BY GATE VOLTAGE FLUCTUATIONS AND GATE CALIBRATION}

More relevant than the speed of a quantum computer is the ratio of the time required for logical operation to the decoherence time of the qubits. If this ratio is less than $\sim 10^{-4}$, then perfect error correction becomes possible [8] [7]. The long relaxation times noted above for Si:P were measured in bulk samples with very low doping density. In the computer architecture, however, the spins are located near a surface and beneath metallic gates, which will inevitably introduce additional decoherence mechanisms for the spins. While the degree of degradation of the spin relaxation rates will need to be determined by experiments, it is worthwhile to estimate the decoherence introduced by thermal fluctuations of the gate voltages on the spins. The simplest case to treat is the effect of voltage fluctuations on $A$-gates on the spin beneath them. The spin is essentially a voltage controlled oscillator, and voltage fluctuations lead to phase errors (Fig. $6)$. For a white noise spectrum of voltage fluctuations with spectral density $S_{V}$ the dephasing rate is:

$$
t_{\phi}^{-1}=\pi^{2} \alpha^{2}(V) S_{V}
$$

where $\alpha \equiv d \nu / d V$ is the tuning parameter of the VCO. From Fig. 3, it is clear that $\alpha$ can be arbitrarily small if the $A$-gate is biased appropriately. To obtain a crude estimate of $t_{\phi}^{-1}$, I assume $\alpha=100 \mathrm{MHz}$ Volt $^{-1}$. The Johnson noise on a $50 \Omega$ transmission line at room temperature is $S_{V} \cong 10^{-18} \mathrm{~V}^{2} \mathrm{~Hz}^{-1}$. These values give $t_{\phi}^{-1}=0.1 \mathrm{sec}^{-1}$, allowing many thousands of logical operations to be performed in the decoherence time of the computer, and

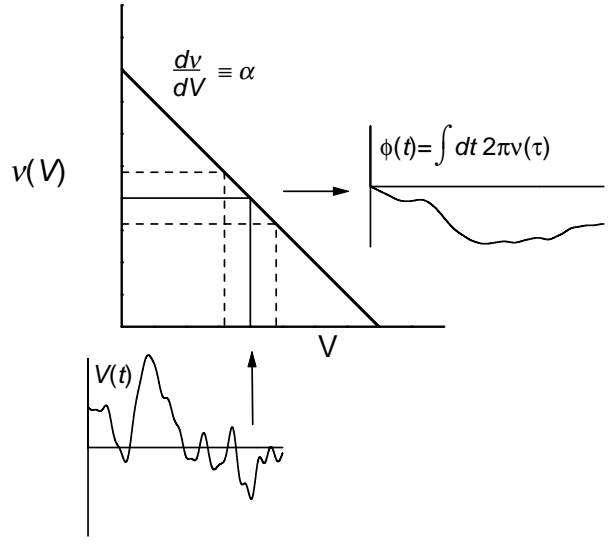

FIG. 6. Since gate voltages are used to control the dynamics of qubits in the proposed architecture, fluctuations in gate bias must inevitably be a source of decoherence. An $A$-gate over a donor can be modeled as a classical voltage-controlled oscillator. The larger the VCO tuning parameter $\alpha$ the more rapidly phase error in the spin state builds up with time.

indicating that Si based nuclear spin quantum computers are potentially in the realm where continuous error correction is possible.

A more serious issue arises from low frequency fluctuations ( $1 / f$ noise) of voltages on the gates and of electric fields at the donors arising from charge motion within the semiconductor host. As $f \rightarrow 0$ these fluctuations become variable offsets of the gates that must be calibrated for the computer to operate properly. In principle each of the gates in the computer can be individually calibrated by performing simple measurements on the operation of each gate. Clearly, however, this process will become increasingly cumbersome as the size of the computer is scaled up, particularly if regular recalibration is necessary. A computer architecture which does not require calibration of individual gates, and is consequently resilient in the presence of low frequency fluctuations, is discussed below.

\section{TECHNIQUES FOR SINGLE SPIN MEASUREMENT}

The procedures outlined above perform all of the logical operations necessary for quantum computation. The spins must also be prepared in a specified initial state and read out at the end of the calculation. In a computer architecture capable of performing error correction, state preparation and measurement are necessary throughout the process of calculation. For this to be pos- 
sible the measurement must be rapid, since if the measurement process during error correction were slow, the qubits would decohere during the period in which the measurement was occurring. While the measurement of the magnetic fields induced by electron and even nuclear spins may be possible using advanced magnetic resonance force microscopy [26] [27], these techniques are highly unlikely to perform measurements on a time scale comparable to logical operations. While single spin measurements are likely to be both difficult and slow, single charge measurements on a microsecond time scale are now routinely performed using single electron transistor (SET) electrometers [28], and SET's may be ideally suited to perform the quantum measurements necessary for quantum computation 29.

Probably the simplest system in which spin to charge conversion is possible is a system of two electrons in a common potential well. The Pauli Principle forbids both electrons from being in the same orbital state unless they have opposite spins and are in a singlet state. Consequently, in the absence of a magnetic field the lowest energy state of a two electron system is a spin singlet, and triplet states are higher in energy 30]. An electrometer capable of detecting the number of electrons occupying a bound state can thus determine whether two electrons are in a singlet or triplet state.

Measurement of a single electron spin requires that one of the two electrons be in a known spin state, for example, $\mid \downarrow$ ?〉. (Techniques for preparing electrons in known spin states will be discussed below.) If the state of the system is $|\downarrow \downarrow\rangle$ then a measurement of the two electrons will yield a triplet result. If the two electrons are in the $|\uparrow \downarrow\rangle$ state, then they may be either in a singlet $(|\uparrow \downarrow-\downarrow \uparrow\rangle)$ or a triplet $(|\uparrow \downarrow+\downarrow \uparrow\rangle)$ state. Scattering between $|\uparrow \downarrow-\downarrow \uparrow\rangle$ and $|\uparrow \downarrow+\downarrow \uparrow\rangle$ is generally much more rapid then scattering between $|\downarrow \downarrow\rangle$ and $|\downarrow \uparrow\rangle$, since scattering between the latter states can only proceed via a spin flip. Consequently, a measurement which determines whether the electrons are in a singlet or triplet state can be used to infer the spin state of the second electron if the measurement time exceeds the time required for scattering between the $|\uparrow \downarrow-\downarrow \uparrow\rangle$ and $|\uparrow \downarrow+\downarrow \uparrow\rangle$ states but is less than the time required for scattering between $|\downarrow \downarrow\rangle$ and $|\downarrow \uparrow\rangle$.

The potential well binding the two electrons can be provided by a donor state in Si. A singly charged donor site, such as $\mathrm{Si}: \mathrm{P}$ is capable of binding two electrons to form a $D^{-}$state only if the electrons are in a singlet state. The binding energy of this state is small, however, and probing it will be difficult 31 32. Doubly charged donors, such as Column VI impurities in Si, have strongly bound two electron states. In $\mathrm{Si}$ Te, the singlet ground state is almost $150 \mathrm{meV}$ below the lowest lying triplet state [33] [34]. Column VI donors also have stable isotopes with zero nuclear spin, allowing two electron systems to be probed in the absence of an additional hy- a)

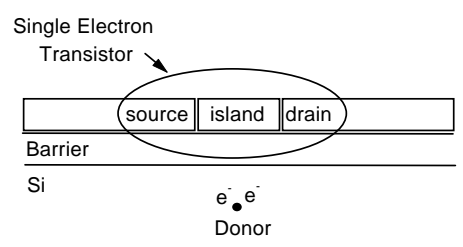

b)

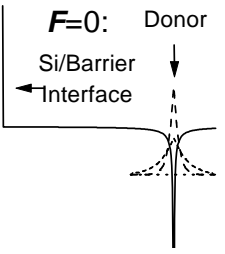

c)

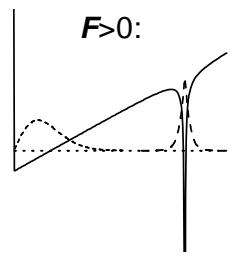

FIG. 7. (a) Possible configuration in which a single electron transistor (SET) is used as a sensitive electrometer to probe the spin state of a two-electron system. The conduction across a SET depends sensitively on the potential of the island electrode. (b) In the absence of an applied electric field $F$ the wavefunctions of both electrons (dotted lines) are localized by the attractive electrostatic potential (solid line) in the vicinity of the donor. (c) An applied electric field can draw one of the two electrons to a state at the Si-barrier interface. The value of $F$ where this electron moves from the interface to the donor is different for electrons in singlet and triplet spin states. This charge motion affects the SET conductance, enabling the spin state of the electrons to be determined.

perfine interaction with the donor nucleus.

An experimental configuration in which a two electron system is probed by a SET is shown in Fig. 7. The SET island lies directly above a donor embedded in $\mathrm{Si}$, which is separated from the SET by a barrier layer. A potential bias between the island and the substrate is capable of ionizing the donor and drawing one of the two electrons to a state at the $\mathrm{Si} /$ barrier interface. Charge motion between the interface state and the donor state will change the potential of the SET island and hence the conductance of the SET. Because the binding energy of the donor is different for singlet and triplet spin states, detection of charge motion onto the impurity will allow the spin state of the electrons to be determined.

For an rf-SET 28, well coupled to a Si:Te donor, a measurement time of order microseconds has been estimated [35], much smaller then the expected spin flip scattering time $(|\downarrow \downarrow\rangle \leftrightarrow|\downarrow \uparrow\rangle)$ of the system. It may also be possible to measure the spin state using a more conventional FET as an electrometer [5]. Clearly experiments will be necessary to determine the actual speed of measurement and the scattering times in real systems.

Because electron and nuclear spins are coupled by the hyperfine interaction, polarization transfer between electrons and nuclei are possible. Consequently, a nuclear spin can be measured by allowing it to interact with a 
two electron system in a known spin state, and subsequently measuring the electrons using the procedure discussed above.

\section{POSSIBLE IMPROVEMENTS TO THE ARCHITECTURE}

The quantum computer architecture outlined above shows that quantum computation and single spin measurement in Si nanostructures are theoretically possible using devices that are not extraordinarily different from those being currently fabricated. Nonetheless there are several deficiencies of the architecture that may prevent it from being a practical one, and several improvements on the original design have been suggested. I will focus below on some obvious shortcomings: (1) the computer must operate at extremely low temperatures and in very high DC magnetic fields, (2) quantum logic is only possible between nearest neighbor spins, and these spins must be extremely close together $(\sim 100 \AA)$ for the computer to operate, and (3) logical operations must be performed with a precision far exceeding that which is typically obtainable in solid state devices in order to make continuous computation possible. I will discuss below possible modifications to the original architecture which may alleviate the difficulties associated with each of these problems.

\section{A. Spin Refrigeration}

While the computer must operate at extremely low temperatures and in high magnetic fields to fully polarize the electrons, a more moderate environment $(T=1-4$ $\mathrm{K}$ ) would vastly simplify the construction of a computer with many components, when power dissipation will inevitably become a factor. Operation at smaller magnetic fields would also be desirable, firstly since many conventional semiconductor components, which may need to be placed near the computer (or even on the same $\mathrm{Si}$ chip), are rendered inoperative in large magnetic fields. Secondly, matrix elements connecting hyperfine-coupled nuclear spin states increase with reduced magnetic field (Fig. 1c) and operation of the computer can potentially proceed more rapidly at lower magnetic fields. Finally, altogether different quantum computer architectures may be possible as $B \rightarrow 0$.

While $T=100 \mathrm{mK}$ is necessary to fully polarize the electron spins in large laboratory magnetic fields, the spinlattice relaxation time is extremely long even at $T \approx 4$ $\mathrm{K}$, where $t_{1}$ of electrons on donors still exceeds $1 \mathrm{sec} 15$ [16]. Consequently it is relatively easy to create a situation where the spin temperature is much different from the lattice temperature. Construction of a 'spin refrigerator' would create highly polarized spins at more modest lattice temperatures and magnetic fields.

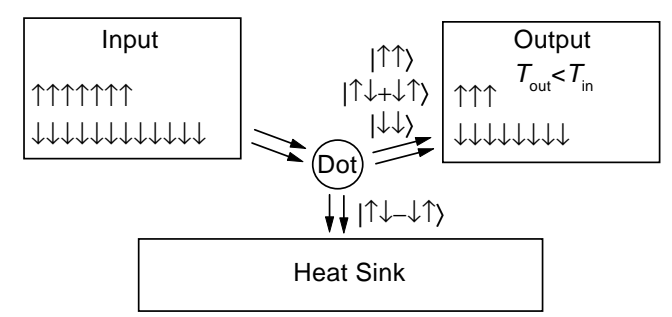

FIG. 8. Devices which can sort singlet and triplet states of electron pairs can be used to refrigerate spins. In the figure, randomly selected pairs of electrons from a partially polarized input reservoir pass through a dot. Singlet pairs are expelled into a heat sink, while triplet pairs pass through to an output reservoir. Because the polarization of the singlet pair is exactly zero, the polarization of the output reservoir must be greater than in the input reservoir and consequently $T_{\text {out }}<T_{\text {in }}$.

Possible methods to introduce nonequilibrium polarizations of the electrons include injection of spins from ferromagnetic contacts and optical pumping. Neither of these techniques alone, however, is likely to achieve the extremely high polarizations necessary for large-scale quantum computation. What would be desirable is an on chip, closed cycle, spin refrigeration device which could increase electron spin polarization.

A possible realization of such a device using the ideas of single spin measurement discussed above is depicted in Fig. 8. Electrons in a partially polarized 'input' reservoir proceed in pairs through a 'dot' and into an 'output' reservoir. On the dot a measurement is performed which distinguishes triplets from singlets. Triplet states are passed through the dot into the output, while singlet pairs are expelled into a heat sink. Because singlet pairs have exactly zero spin polarization, the output reservoir must have higher average polarization than the input reservoir, and hence lower temperature. Such 'singlet rejection refrigeration devices' could be cascaded to produce the high polarizations necessary for quantum computation starting from a modestly polarized input, created either thermally or by injection from a ferromagnet. A circulating bath of cooled polarized electrons could polarize the nuclei of a quantum computer via the hyperfine interaction to initialize qubits and provide the ancilla spins necessary for error correction and measure- 
ment.

Very recently, an approach to quantum computing has been developed based solely on controlling exchange interactions between spins to perform logical operations on qubits encoded on several spins [36]. Using this approach quantum operations would not require magnetic fields, and spin refrigeration would enable the computer to operate at $B \rightarrow 0$.

\section{B. Use of Electron Spins as qubits for transport of quantum information}

In the quantum computer architecture presented above nuclear spin qubits are coupled to each other via indirect exchange processes mediated by the electrons, and the spatial separation between spin qubits cannot greatly exceed the Bohr radius of the donor impurities. Recent experiments [37] clearly show, however, that electron spins can effectively transmit quantum information over much larger distances $(\simeq 100 \mu m)$. While it is important to note that a free electron at a Si interface will have altogether different (and generally shorter) relaxation times than those for an electron bound to a donor, transmitting quantum information using a free electron [9] has the obvious advantage that the donor nuclei being coupled can be separated over much larger distances than the Bohr radius. Quantum information transport by free electrons could also enable quantum computers to have separated, specialized devices for logic, memory, and measurement. Thus, for example, Te donors below SET's could be optimized for measurement operations, while $\mathrm{P}$ donors below $A$-gates could be optimized for quantum logic.

A gated structure in which quantum logic between nuclear spin qubits is mediated by a free electron spin is shown in Fig. 9. First, quantum information is swapped between a donor nuclear spin and an electron bound to the donor by an appropriate rf pulse. The electron is then ionized from the donor by an electric field applied to the gate above the donor and is transmitted to a second donor site through one or more intermediary gates which move the electron along the interface. The electron is then allowed to combine with the second donor and a second SWAP operation is applied to exchange information between the electron and nuclear spin. While the architecture shown in Fig. 9 is similar to that depicted in Fig. 4, the gate dimensions need not be comparable to the donor Bohr radius.

Quantum information in this scenario must be transmitted through a single electron spin. If the mediating electron couples to other electrons through the exchange interaction, then quantum information will be lost. Thus the gate electrodes moving the electron from site to site must store a single electron at a time, and the architecture strongly resembles a single electron charge coupled device (CCD) 38. Also, the interface along which the

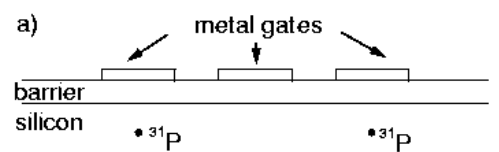

b)

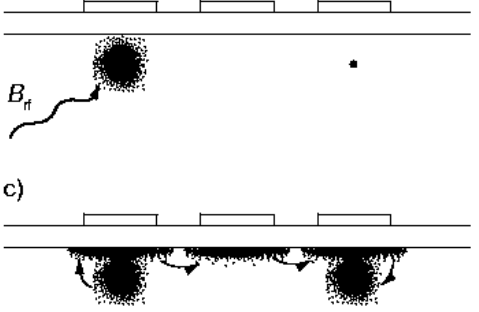

d)

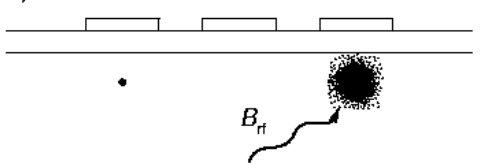

FIG. 9. (a) Possible architecture for quantum logic in which quantum information is transmitted by free electrons. (b) An rf pulse performs an operation which swaps the spin state of the nucleus and the donor electron. (c) The electron is then ionized from the donor and shuttled to a different donor site by applying biases to gates in a manner similar to that used in a charge coupled device. (d) Once it is bound to the second donor, a second rf pulse exchanges spin between the electron and the donor nucleus, completing transfer of quantum information between spins. In this architecture donor spacing can be much larger than in approaches which use exchange coupling between donors for quantum information transfer. 


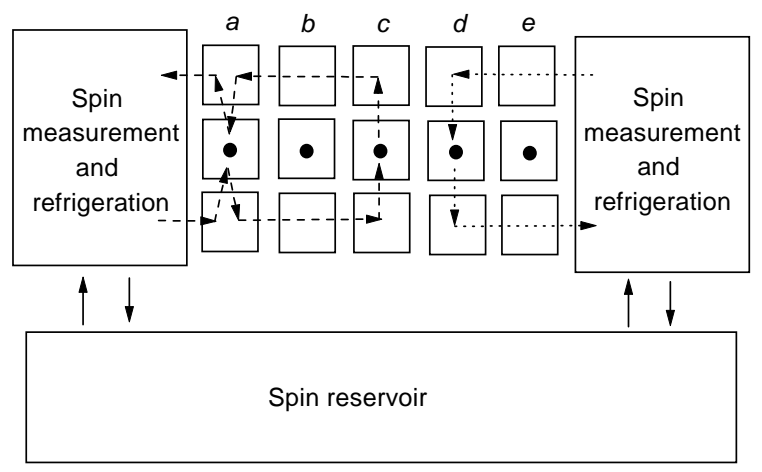

FIG. 10. Drawing of a five qubit quantum computer using gates (squares) to transfer unbound electrons between nuclear spin qubits (black circles). Measurement/refrigeration devices are adjacent to the qubits. Dashed path is for an electron which performs a two qubit logical operation on spins $a$ and $c$. An electron following the dotted path performs a measurement operation on spin $d$. Note that all donor sites and gates do not need to be functioning to do quantum operations on the computer.

electron is transmitted must be almost completely free of trapped charges. A possible quantum computer architecture using these types of devices is shown in Fig. 10. Gate electrodes shuttle single electrons between donors to perform quantum logic and between donors and electron spin measurement devices for measurement of the qubits. The measurement devices also provide a source of polarized spins for qubit initialization and error correction. Such an architecture can be made defect tolerant [39], since all gates and donor sites do not need to be functional in order to do quantum operations and measurement.

Using electron spins to transmit quantum information over relatively large distance will have obvious advantages in terms of implementing quantum algorithms. While quantum computation [10] and error correction [8] are possible using only local gates, efficiency will undoubtedly improve if quantum information can be rapidly transmitted between remote qubits.

\section{Modified RF pulses for uniform gate operations}

Decoherence can be produced in a quantum system not only by interactions of the qubits with uncontrolled degrees of freedom but also by errors in the logical operations on the qubits. Consequently, the accuracy of gate operations in a quantum computer will need to be $\sim 10^{-4}$ to be in the regime in which error correction will enable continuous quantum computation. This level

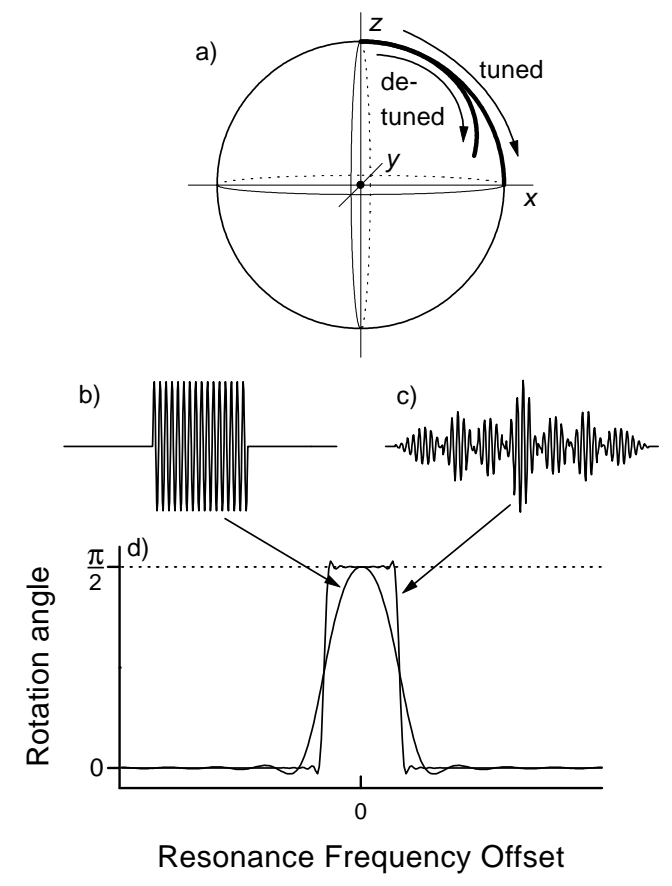

FIG. 11. (a) Illustration of the Bloch sphere of a single qubit. An rf pulse (b) that performs a $\left(\frac{\pi}{2}\right)_{y}$ operation on a qubit resonant with the pulse frequency will perform a different operation on a nonresonant qubit. Modification of the rf pulse shape (c) can compensate for these errors in the neighborhood of the resonance to very high accuracy, however (d).

of precision will be an extremely difficult requirement to meet in any solid state material, where fluctuations of device properties are inevitable. One possible solution is to perform precise calibration of each individual gate in the computer, an approach which will become increasingly difficult in large computers and will be even more arduous if the calibrations themselves fluctuate. A more attractive possibility pointed out by Benjamin [10] is to design gate operations which are highly precise despite device fluctuations.

An example of a fluctuation-tolerant approach to single spin rotations is shown in Fig. 11. A rotation induced by an rf pulse will deviate from the desired value if the resonance frequency of the qubit deviates from the frequency of the pulse. If the rf pulse is shaped appropriately, however, the errors associated with small resonance frequency offsets can be drastically reduced. Shaped and composite pulse waveforms for single spin rotations have been developed to improve accuracy of NMR spectroscopy 40 41] and of bulk spin resonance quantum logic [42]. Extension of these ideas to two qubit operations - while presumably possible in principle - has not yet been addressed. In the computer architecture shown in Fig. 9, different types of rf pulses are used to perform both one and two qubit operations, and shaped 
pulses will allow accurate logical operations to be performed despite appreciable device fluctuations. Because the effect of a shaped pulse is independent of the exact resonance frequency of the qubit, low frequency fluctuations of the resonance frequency would not degrade the accuracy of the logical operations of the computer, and the computer could consequently be highly immune to the inevitable $1 / f$ noise present in solid state materials.

Another problem with the original architecture is that each gate is a channel coupling to the system that can be a source of decoherence [10. While this decoherence is an inevitable byproduct of using gates to control logical operations, the magnitude of the decoherence varies with applied gate bias. For example in Fig. 3 gate-induced decoherence is largest when $\alpha$ is largest, near $V=V_{1}$, while it is much smaller when $\alpha \rightarrow 0$ at $V=V_{0}$ and $V=V_{2}$. Shaped pulses could enable "digitally gated" quantum computer architectures in which the gates are biased only at two low decoherence values, one resonant and one nonresonant with the applied $B_{r f}$. Gates associated with idling qubits are biased at their nonresonant value. A logical operation on a spin is performed by rapidly bringing the gate above the spin to its resonant voltage and applying an rf pulse. At the completion of the pulse, the gate is rapidly brought back to its nonresonant setting. In this manner the gates still allow selective operations to be performed on the qubits without introducing decoherence, except during the brief periods when the gates are switched.

Even if shaped pulses are used for quantum logic, it is likely that some degree of calibration will be necessary in any solid state quantum computer. While this may appear to be a fundamental obstacle to large-scale quantum computation, it is important to remember that the classical computational resources associated with the operation of any quantum computer can be large, and consequently a large amount of conventional computer memory and processing power can be devoted to the proper calibration of each gate.

\section{IMPLEMENTING THE ARCHITECTURE IN $\mathrm{Si} / \mathrm{Si}_{x} \mathbf{G e}_{1-x}$ HETEROSTRUCTURES}

In all the device structures discussed above, a barrier material must be present separating the Si containing the donors from the conducting gates. The Si/barrier interface must be almost entirely free of charge and spin defects if the devices are to perform quantum gate operations, especially if free electrons on the interface are to be used to transfer quantum information between remote donors. Silicon oxide and nitride layers - used in conventional MOS structures - are amorphous materials, and their interfaces with Si will inevitably contain charge centers associated with dangling bonds, rendering their use in a quantum computer highly problematic

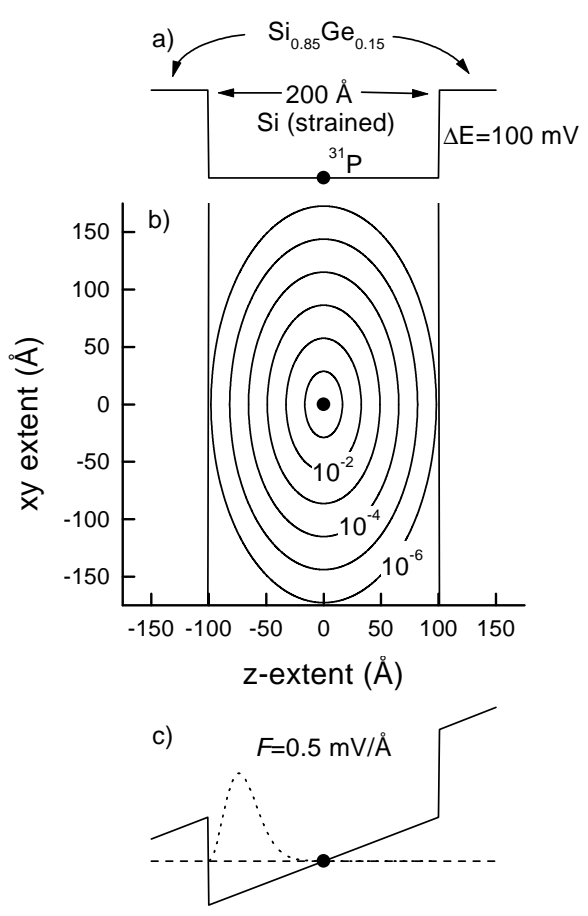

FIG. 12. (a) Possible realization of the quantum computer architecture where the electron and the donor reside in a strained Si quantum well surrounded by unstrained $\mathrm{Si}_{x} \mathrm{Ge}_{1-x}$. (b) Because of the strain, the electron mass for motion parallel to the layers is smaller than the mass for motion perpendicular to the layers, and the contours of equal probability of the electron wavefunction are ellipsoidal. (c) An applied electric field $F$ can draw the electron to an interface state confined in the well.

[43. Various epitaxial barrier materials on Si have been developed, however, that may ultimately have the extraordinarily low defect densities that will be necessary in a quantum computer.

Several oxide materials, including $\mathrm{CeO}_{2}$,44] and $\mathrm{SrTiO}_{3}$ [45, are being explored which may grow epitaxially on pure Si. Most promising for application to quantum computation, however, are the heterostructures grown with Group IV elements: $\mathrm{Si} / \mathrm{Si}_{x} \mathrm{Ge}_{1-x}$ [5] and $\mathrm{Si} / \mathrm{Si}_{x} \mathrm{C}_{1-x}$. I will focus on $\mathrm{Si} / \mathrm{Si}_{x} \mathrm{Ge}_{1-x}$ heterostructures because they are the most technologically developed. $\mathrm{Si} / \mathrm{Si}_{x} \mathrm{C}_{1-x}$ heterostructures have recently been used to perform the first direct electron spin resonance measurements [46] [17] on two dimensional electron systems (2DES), and they may thus also be relevant for implementing quantum logical devices.

Because of the lattice mismatch between $\mathrm{Si}$ and Ge, $\mathrm{Si}_{x} \mathrm{Ge}_{1-x}$ heterostructures must inevitably contain layers that are strained 48. A high quality (mobility $500,000 \mathrm{~cm}^{2} /$ Vsec 49]) 2DES has been formed in these 


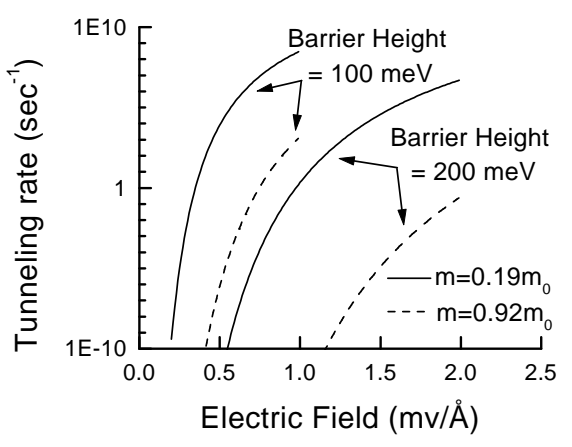

FIG. 13. Fowler-Nordheim tunneling rates for electrons out of a strained $\mathrm{Si}$ quantum well through a $\mathrm{Si}_{x} \mathrm{Ge}_{1-x}$ barrier. $\mathrm{Si} / \mathrm{Si}_{x} \mathrm{Ge}_{1-x}$ structures for quantum logic will need to be carefully designed so that electron tunneling out of the well is not a significant source of decoherence.

materials by confining the electrons in a strained Si layer between unstrained layers of $\mathrm{Si}_{x} \mathrm{Ge}_{1-x}$ on a (100) oriented substrate (Fig. 12a). Because the $\mathrm{Si}$ is strained along a (100) axis, the valley degeneracy of the conduction band is broken, and the well is occupied by electrons only in the two valleys with minima on the axis perpendicular to the layers. The electron effective mass in these valleys is anisotropic [50], and the contours of equal probability density of electron wavefunctions on donors in the Si well are ellipsoidal (Fig. 12b).

Since the Si layer is under strain, its thickness cannot exceed a critical value without the nucleation of dislocations 48]. For $\mathrm{Si} / \mathrm{Si}_{0.85} \mathrm{Ge}_{0.15}$ this thickness is approximately $200 \AA$, sufficiently thick so that the electron wavefunction of a donor in the center of the well is almost entirely in pure Si.

Conduction band offsets in $\mathrm{Si} / \mathrm{Si}_{x} \mathrm{Ge}_{1-x}$ are not large, and consequently electron tunneling through the barrier is a potential source of decoherence. Estimates of the Fowler-Nordheim tunneling rate [51] depend strongly on the electron mass, however (Fig. 13). While tunneling out of the well is in the direction of the heavier Si electron mass, coupling between valleys may be large enough that tunneling rates will be intermediate between those calculated for the two electron mass values. Experiments will be necessary to determine electron tunneling rates before $\mathrm{Si} / \mathrm{Si}_{x} \mathrm{Ge}_{1-x}$ devices for quantum computing can be optimized. For $x=0.85$ the depth of the well is about $100 \mathrm{meV}$, probably sufficient to allow the application of
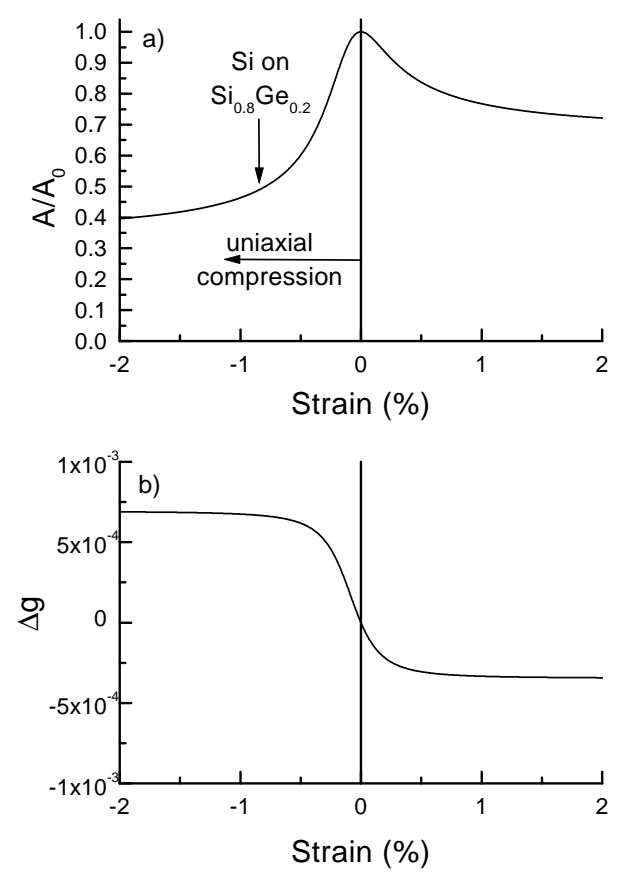

FIG. 14. The effect of strain on the hyperfine interaction frequency (a) and the $g$-factor anisotropy (b) in Si, using calculations in Ref. 52]. In the strained layer stucture shown in Fig. 12, the hyperfine interaction will be reduced about $50 \%$ from the value observed in unstrained $\mathrm{Si}$.

large enough electric fields to ionize the donor (Fig. 12c) without excessive tunneling leakage.

Straining the Si layer also reduces the hyperfine interaction energy $A$ and the $g$-factor anisotropy of the coupled electron-nuclear spin states (Fig. 14) [52]. In the strained well $A$ will be approximately $50 \%$ of its value in unstrained Si. Also relevant is that fluctuations in static strain (created during device fabrication for example) will affect the values of the resonance frequencies. Fluctuations of the $g$ factor caused by strain are actually reduced in highly strained layers, however.

\section{DOPANT INTRODUCTION BY LOW ENERGY ION IMPLANTATION}

Finally, I consider possible approaches to introducing single donor ions in controllable locations into the Si. The low density of unwanted impurities introduced into the $\mathrm{Si}$ that is required for the computer suggests that both crystal growth and dopant incorporation should be performed in ultra high vacuum in the same apparatus. Possible approaches to dopant placement include nanoassembly using a scanned probe and ion implantation [5]. At 
the temperatures necessary for optimal growth of high quality material [49], surface segregation of donors deposited onto a Si surface is substantial [53], leading to large $(\geq 100 \AA)$ vertical (and presumably lateral) displacements of deposited donors from their original location. The diffusion of donors once they are incorporated into the bulk is, however, much lower. Low energy $(\simeq 150$ $\mathrm{eV}$ ) ion implantation has consequently been developed as a tool for introducing donors into semiconductors with extremely sharp $(\leq 30 \AA)$ vertical profiles [54]. A tool which could place donors into $\mathrm{Si}$ with the precision necessary for development of the proposed quantum computer would be a low energy focused ion beam with single ion implantation capability [55] incorporated into a Si MBE growth chamber. The development of such a tool would likely be a significant technological challenge, and many important experiments can first be done to assess the viability of the proposed architecture with simpler techniques and equipment.

\section{PROSPECTS AND CONCLUSIONS}

The obstacles to building a silicon-based quantum computer are enormous, and much research needs to be done before even the viability of the ideas presented above can be determined. Priorities in this research must be: (1) the fabrication of single spin measurement devices, (2) measurement of spin decoherence mechanisms of electrons and nuclei at donors and of free electrons in real Si heterostructure materials, (3) the development of single charge CCD-like devices capable of transporting quantum information on an electron spin, (4) the design of quantum logical operations which are tolerant to the fluctuations inevitable in solid state materials, and finally (5) the demonstration of a technology for placing single donors into a semiconductor substrate at precisely specified locations.

Single spin measurement devices (and also presumably spin refrigerators) will be of interest in their own right, irrespective of their impact on quantum computation. It is likely that they can be fabricated with currently available technology [35], and they can subsequently be applied to the detailed measurement of decoherence in real nanostructure devices. The development of single charge CCD's will be an important milestone showing that the extreme material purity necessary for the proposed quantum computer architecture is achievable.

Large-scale quantum computers will need to have fluctuation and defect tolerant designs, both because of the precision that is required for doing quantum logic and because of the inevitable variations in solid state materials from device to device. The technology for donor placement which will need to be developed may also have applications outside of quantum computation, since fluctuations attributable to the random locations of donors are already an issue for ultra-small conventional semiconductor devices.

Because technological advances need to be made on many fronts, it is currently not possible to ascertain whether the architecture for Si-based quantum computers presented above is viable. The outcome of research in the next few years focused on the issues mentioned above, however, will determine whether silicon - the material used in today's computers -is also capable of being the host material for a revolutionary new type of machine.

\section{ACKNOWLEDGEMENTS}

The author has benefited from discussions with S. Barrett, X. Hu, G. Lucovsky, S. Lyon, J. Melngailis, R. Schoelkopf, P. Thompson, and E. Yablonovich.

[1] D. Loss and D. P. DiVincenzo, Phys. Rev. A 57, 120 (1998).

[2] V. Privman, I. D. Vagner, and G. Kventsel, Phys. Lett. A 239, 141 (1998).

[3] B. E. Kane, Nature 393, 133 (1998).

[4] A. Imamoglu et al., Quantum information processing using electron spins and cavity-QED, quant-ph/9904096.

[5] R. Vrijen et al., Electron spin resonance transistors for quantum computing in Silicon-Germanium heterostructures, quant-ph/9905096.

[6] S. Bandyopadhyay, A self assembled nanoelectronic quantum computer based on the Rashba effect in quantum dots, quant-ph/9910032.

[7] E. Knill, R. Laflamme, and W. H. Zurek, Science 279, 342 (1998).

[8] D. Gottesman, Fault-tolerant quantum computation with local gates, quant-ph/9903099.

[9] D. P. DiVincenzo and D. Loss, J. of Magnetism and Magnetic Matls. 200, 202 (1999).

[10] S. C. Benjamin, Phys. Rev. A 61, 020301 (2000).

[11] W. Kohn, in Solid State Physics, edited by F. Seitz and D. Turnbull (Academic, New York, 1957), Vol. 5, pp. 257-320.

[12] C. P. Slichter, Principles of Magnetic Resonance, 3rd ed. (Springer-Verlag, Berlin, 1990), Chap. 4.

[13] R. Feynman, The Feynman Lectures on Physics (Addison-Wesley, Reading Massachusetts, 1965), Vol. 3, Chap. 12.

[14] G. Feher, Phys. Rev. 114, 1219 (1959).

[15] G. Feher and E. A. Gere, Phys. Rev. 114, 1245 (1959).

[16] A. Honig and E. Stupp, Phys. Rev. 117, 69 (1960).

[17] J. P. Gordon and K. D. Bowers, Phys. Rev. Lett. 1, 368 (1958).

[18] M. Chiba and A. Hirai, J. Phys. Soc. Japan 33, 730 (1972). 
[19] L. Viola, S. Lloyd, and E. Knill, Phys. Rev. Lett. 83, 4888 (1999).

[20] D. P. DiVincenzo, Science 270, 255 (1995).

[21] K. A. Valiev and A. A. Kokin, Solid state NMR quantum computer with individual access to qubits and some of its ensemble developments, quant-ph/9909008.

[22] C. Herring and M. Flicker, Phys. Rev. 134, A362 (1964).

[23] G. Burkard, D. Loss, and D. P. DiVincenzo, Phys. Rev. B 59, 2070 (1999).

[24] X. Hu and S. D. Sarma, Hilbert space structure of a solid state quantum computer: two-electron states of a double quantum dot artificial molecule, quant-ph/9911080.

[25] K. Andres et al., Phys. Rev. B 24, 244 (1981).

[26] J. A. Sidles et al., Rev. Mod. Phys. 67, 249 (1995).

[27] K. Wago et al., Rev. Sci. Instrum. 68, 1823 (1997).

[28] R. J. Schoelkopf et al., Science 280, 1238 (1998).

[29] A. Shnirman and G. Schön, Phys. Rev. B 57, 15400 (1998).

[30] N. Ashcroft and D. Mermin, Solid State Physics (Saunders College, Philadelphia, 1976), Chap. 32.

[31] D. M. Larsen, Phys. Rev. B 23, 5521 (1981).

[32] D. M. Larsen and S. Y. McCann, Phys. Rev. B 46, 3966 (1992).

[33] H. G. Grimmeiss and E. Janzen, in Deep Centers in Semiconductors, edited by S. Pantelides (Gordon and Breach, New York, 1986), Chap. 2.

[34] G. Grossmann, K. Bergman, and M. Kleverman, Physica B 146, 30 (1987).

[35] B. E. Kane et al., Phys. Rev. B 61, 2961 (2000).

[36] D. Bacon, J. Kempe, D. A. Lidar, and K. B. Whaley, Universal fault-tolerant quantum computation on decoherence-free subspaces, quant-ph/9909058.

[37] J. M. Kikkawa and D. D. Awschalom, Nature 397, 139 (1999).

[38] S. M. Sze, Physics of Semiconductor Devices, 2nd ed. (Wiley-Interscience, New York, 1981), Chap. 7.

[39] J. R. Heath, P. J. Kuekes, G. S. Snider, and R. S. Williams, Science 280, 1716 (1998).

[40] H. Geen and R. Freeman, Journal of Magnetic Resonance 93, 93 (1991).

[41] R. Freeman, Spin Choreography (Spektrum, Oxford, 1997), Chap. 5.

[42] H. K. Cummins and J. A. Jones, Use of composite rotations to correct systematic errors in NMR quantum computation, quant-ph/9911072.

[43] G. Lucovsky et al., Appl. Phys. Lett. 74, 2005 (1999).

[44] J. T. Jones et al., J. Vac. Sci. Technol. B 16, 2686 (1998).

[45] R. A. McKee, F. J. Walker, and M. F. Chisholm, Phys. Rev. Lett 81, 3014 (1998).

[46] N. Nestle et al., Phys. Rev. B 56, 4359 (1997).

[47] H. J. Kümmerer et al., Phys. Rev. B 59, 12568 (1999).

[48] S. C. Jain, Germanium-Silicon Strained Layers and Heterostructures (Academic Press, Boston, 1994).

[49] K. Ismail et al., Appl. Phys. Lett. 66, 1077 (1995).

[50] T. Ando, A. B. Fowler, and F. Stern, Rev. Mod. Phys. 54, 437 (1982).

[51] S. Nagano et al., J. Appl. Phys. 75, 3530 (1994).

[52] D. K. Wilson and G. Feher, Phys. Rev. 124, 1068 (1961).

[53] K. D. Hobart, F. J. Kub, G. G. Jernigan, and P. E. Thompson, J. Vac. Sci. Technol. B 14, 2229 (1996).

[54] P. Fons et al., Appl. Phys. Lett. 53, 1732 (1988).
[55] T. Shinada et al., J. Vac Sci. Technol. B 16, 2489 (1998). 